\title{
Robust Reflection Detection and Removal in Rainy Conditions using LAB and HSV Color Spaces
}

\author{
Synh Viet-Uyen Ha, Nhan Thanh Pham, Long Hoang Pham, Ha Manh Tran \\ School of Computer Science and Engineering, International University, Ho Chi Minh City, Vietnam
}

Correspondence: Synh Viet-Uyen Ha, hvusynh@hcmiu.edu.vn

Communication: received 15 December 2015, revised 29 March 2016, accepted 4 April 2016

Online publication: 24 August 2016, Digital Object Identifier: 10.21553/rev-jec.130

The associate editor coordinating the review of this article and recommending it for publication was Dr. Le Vu Ha.

\begin{abstract}
In the field of traffic monitoring systems, shadows are the main causes of errors in computer vision-based vehicle detection and classification. A great number of research have been carried out to detect and remove shadows. However, these research works only focused on solving shadow problems in daytime traffic scenes. Up to now, far too little attention has been paid to the problem caused by vehicles' reflections in rainy conditions. Unlike shadows in the daytime, which are homogeneous gray shades, reflection shadows are inhomogeneous regions of different colors. This characteristic makes reflections harder to detect and remove. Therefore, in this paper, we aim to develop a reflection detection and removal method from single images or video. Reflections are detected by determining a combination of $L$ and $B$ channels from LAB color space and $\mathrm{H}$ channel from HSV color space. The reflection removal method is performed by determining the optimal intensity of reflected areas so that they match with neighbor regions. The advantage of our method is that all reflected areas are removed without affecting vehicles' textures or details.
\end{abstract}

Keywords- HSV, LAB, rainy conditions, reflection detection, reflection removal.

\section{INTRODUCTION}

Shadow detection and removal have always played a crucial role in traffic surveillance systems that are based on computer vision techniques. In this paper, we focus on solving the problems of shadow in rainy conditions. The shadows, in this case called reflections, post two main problems. Firstly, in daytime condition, the cast shadow is normally a uniform gray region. Meanwhile in rainy conditions, the vehicle reflection consists of a variety of colors. This greatly reduces the accuracy of shadow detection algorithms which are solely based on finding the intensity differences between the image background and foreground. Secondly, headlights are also reflected on the road which distort the length and shape of vehicles. Since reflections also have the same motion as the vehicles casting them, hence distort the shapes, sizes, and colors of vehicles. Thus, it can cause significant errors in vehicle detection and classification.

In this paper, we tackle three problems of vehicle reflection: reflection detection, reflection removal, and headlight reflection.

In the case of reflection detection and removal, several methods have been proposed in recent years. Some noticeable works focused on exploiting the change in chromaticity properties of objects and their shadows. Cucchiara et al. [1-3] mainly focused on the changing of light illuminance in HSV color space to detect shadow regions. Fredembach et al. [4] also applied the same idea with near-infrared spectrum of the image which is captured by a special camera sensor. Xiang et al. [5] proposed a local intensity ratio model (LIRM) which is robust to illumination change. Based on the analysis of the illumination and shadow model, the moving shadowless objects are segmented using normalized local intensity ratio via Gaussian mixture model (GMM). Guan et al. [6] presented a robust cast shadow detection algorithm by integrating multiple cues. They first apply a weak shadow detector to detect these potential shadow pixels. Then three adaptive shadow estimators are designed and cascaded to integrate texture, chromaticity, brightness for eliminating the object pixels, and hence can robustly detect the moving cast shadow. Sanin et al. [7] provided techniques that use chromaticity information to create a mask of pedestrian reflection on the floor. Then the gradient information is employed to remove foreground pixels that were incorrectly included in the mask. Conte et al. [8] proposed a reflection detection model that uses both chromatic properties of the reflections and geometrical constraints on their positions. These methods present good result in specific cases, especially in strong intensity environment.

However, in rainy conditions, where light intensity is low, there is no strong difference between shadow pixels and normal ones, these methods failed to distinguish shadow regions from dark objects. $\mathrm{Xu}$ et al. [9] proposed a combined method to remove both vague and hard shadows in a single image. Working in the gradient domain, their system focuses on providing robust shadow edge estimation. The proposed method is remarkably robust and does not require any information about the shadow geometry. However, the recovered image still contains halos and the areas near the shadow edge would have artifacts that look unnatural.

Headlight reflection poses a major problem when vehicles are observered in rainy weather. Despite that a 
search of the literature revealed only a few studies that try to deal with this problem. The headlight detection method used by Cucchiara et al. [10] can detect vehicles' headlights by applying morphological analysis, as well as taking advantage of headlights shape and size information. Zhang et al. [11] detected headlights by computing the reflection intensity map and reflection suppressed map. Then they combined the two maps and applied a Markov Random Field (MRF). Cabini et al. [12] presented a self-adaptive stereo vision extractor of 3D edges for obstacle, and three kinds of vehicle lights are detected using the $L^{*} a^{*} b$ color space. The three types of light are rear lights and rear-brakelights and warning lights, as well as reverse lights and headlights.

In this research, we propose a reflection detection and removal algorithm that can work robustly in rainy conditions using data from actual traffic surveillance video. The proposed algorithm inherits one core idea from the works of Murali et al. [13] and Suny et al. [14], which exploit the LAB color space. We also incorporate the HSV color space mentioned in [1-3] to our method. We will combine information from both LAB color space and HSV color space to detect the reflected areas. This method can provide good results, and hence achieve better accuracy when removing reflection but still maintain vehicles' textures. The reflection removal is performed by gradually scaling the intensity of reflected areas to match with the average value of the best-fit neighbor region. This approach is somewhat similar to the one presented by Salamati et al. [15], in which a probability map is used to increase the lightness of the pixels in the shadow regions. In the proposed method, we use the meanshift algorithm to calculate the average intensity value for each neighbor region and lighten up the shadow parts according to it. Finally, we also propose a simple technique to deal with headlights' reflections. We first investigate the $\mathrm{L}$ channel to locate the highest intensity pixels, which represent the source of headlight and its reflection. Then the headlights with the reflection that has soundable amount of displacement are grouped together. After that we simply remove the bottom segment as it represents the reflection. Comparing to [14], our method can provide a better solution in rainy conditions. Furthermore, the proposed method can run perfectly fine with low quality videos from the surveillance system in Vietnam.

The rest of the paper is organized as follows. Section 2 describes our proposed reflection detection and removal method along with a brief introduction about the preparation steps. Section 3 presents a way to handle the problem of vehicles' headlight reflection. Section 4 provides the experiments, results and discussion, followed by the conclusion.

\section{Reflection Detection and Removal}

\subsection{Vehicle Detection}

The main goal of the proposed method is to segment and separate moving vehicles in surveillance videos.
Table I

Summary of LAB AND HSV COLOR SPACES

\begin{tabular}{ccl}
\hline Channel & Range & \multicolumn{1}{c}{ Description } \\
\hline L & $0-100$ & Lightness of color (Black to White) \\
A & $-128-127$ & Color channel (Green to Magenta) \\
B & $-128-127$ & Color channel (Blue to Yellow) \\
\hline H & $0-360$ & Color channel (Red, Green, Blue in turn) \\
S & $0.00-1.00$ & Color intensity (Dark to Light) \\
V & $0.00-1.00$ & Lightness of color (Black to White) \\
\hline
\end{tabular}

Therefore, static objects are deemed irrelevant and need to be removed. In this paper, background subtraction technique suggested by [16] was used to model the background and produce the moving foreground objects from the input video. Furthermore, we only concentrate on the central lanes facing the camera and reject other areas of the frame. By doing so, we can also achieve a significant improvement in computational cost. In this paper, we use lane detection technique described in the work of Ha et al. [17]. This method first estimates the traffic flows using a probabilistic model suggested by [18]. Then watershed segmentation technique is applied to complete the lane model. This approach has significant advantages over methods that rely heavily on detecting the lane markers on the road. Thus, it can easily overcome the issue of missing or unavailability of lane markers. Figure 1(b) illustrates the extraction of moving vehicles which are represented in binary blobs.

Then, vehicle detection technique, which is described in $[19,20]$, is applied. We first calculate the contour of each foreground object and use the contour size as a mean to filter noise and small objects. After that a bounding box is applied to each vehicle blob. Using the size and location of these bounding boxes, we extract the moving vehicles from the input image into separated vehicle images. This step helps reducing the computation effort since later processes perform only on a smaller set of images. Sub-figures (c), (d), (e) of Figure 1 illustrates the extracted vehicles using vehicle detection technique.

\subsection{Reflection Detection}

When the dataset is ready, we then proceed with the reflection detection algorithm. The main goal of the proposed method is to detect and locate the reflected areas in the input image. A combination of LAB and HSV color spaces is used. In this method, the original RGB input image is cloned and converted to LAB and HSV color spaces. The LAB color space contains a lightness channel (L), and two color channels (A, B). HSV color space contains three channels: $\mathrm{H}, \mathrm{S}, \mathrm{V}$ which represent hue, saturation, and value, respectively. Table I and Figure 2 provide more information about channels of these two color spaces.

Our method in this paper focuses on $\mathrm{L}$ and $\mathrm{H}$ channels. Since the reflected areas are darker because it has the same meaning with "darker", using the information provided in Table I we can effectively use the L channel to locate these areas. However, dark objects 


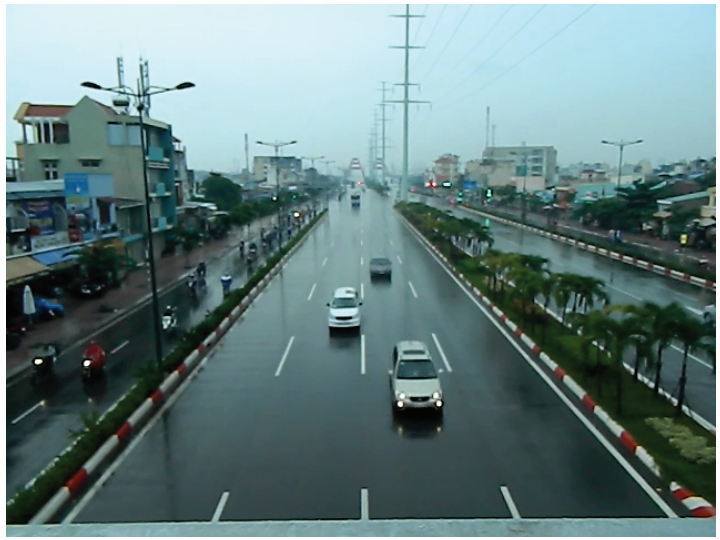

(a)

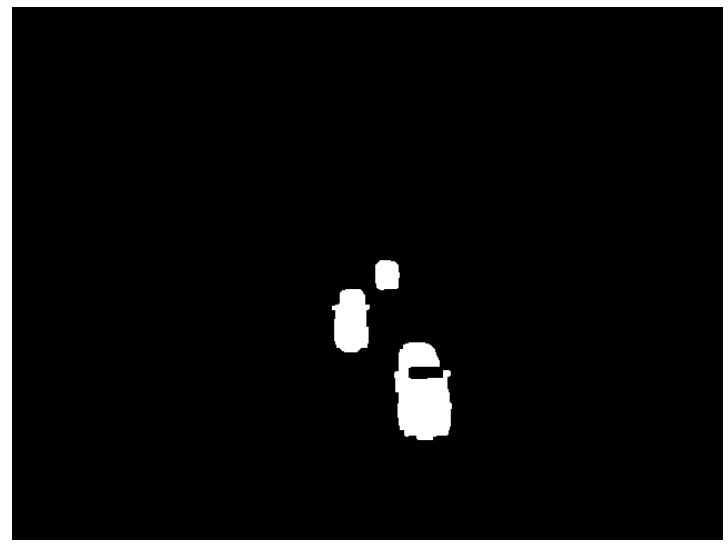

(b)

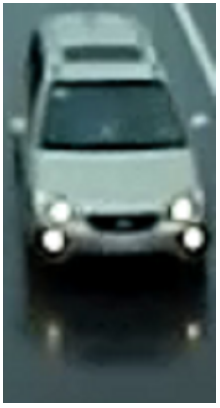

(c)

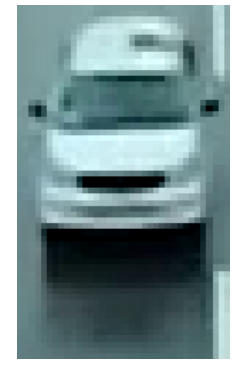

(d)

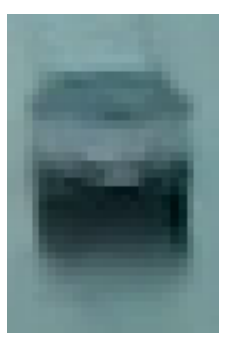

(e)

Figure 1. Results from background subtraction module. (a) original input image, (b) moving foreground objects, (c) extracted vehicle with headlight reflection, (d) extracted vehicle with reflection, (e) extracted vehicle with reflections.

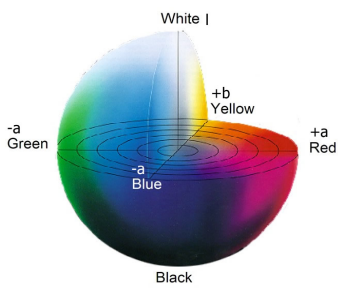

(a)

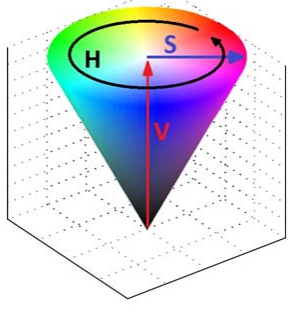

(b)
Figure 2. Illustration of LAB and HSV color model. (a) LAB color space, (b) HSV color space

such as black vehicles share many similar features with reflection. So they can be mistakenly marked as reflection if only $\mathrm{L}$ channel values are considered. To solve this problem, we apply the $\mathrm{H}$ channel. In the $\mathrm{H}$ channel, reflected areas tend to have lower values. By combining the values of $\mathrm{L}$ and $\mathrm{H}$ channels, any pixels that have lower values than a certain threshold are marked as reflection, and others are marked as nonreflection. Particularly, the values of pixels in both $\mathrm{L}$ and $\mathrm{H}$ channels are compared in turned. Let $R$ be the group of reflected pixels; pixel $p$ belongs to $R$ if its lightness value $L(p)$ and hue value $H(p)$ are lower than given thresholds $(\alpha, \beta)$, then

$$
\forall p, p \in R \Longleftrightarrow\left\{\begin{array}{l}
L(p)<\alpha \\
H(p)<\beta
\end{array} .\right.
$$

Based on the results in [12], we adapt the optimal
Table II

Threshold Values for $\alpha$ and $\beta$ Described in Equation (1)

\begin{tabular}{ccc}
\hline Conditions & $\alpha$ & $\beta$ \\
\hline High Intensity & 46 & 54 \\
\hline Low Intensity & 63 & 62 \\
\hline
\end{tabular}

thresholds $\alpha$ and $\beta$ in different sequences, so as to achieve adaptive shadow detection. Table II summarizes the chosen threshold values in this paper.

After pixels are classified, we apply morphological operations to remove isolated pixels. Mis-marked pixels are resolved using dilation and erosion. Then, marked areas which are not large enough (whose number of pixels is smaller than a certain threshold) will be removed. Finally, the remaining areas are classified as reflection. Figure 3 shows that the shadowed areas are successfully detected using the proposed method.

\subsection{Reflection Removal}

After obtaining the location of reflected areas, a method is required to eliminate them. We studied one shadow removal method proposed by Xiang et al. [5]. It removes shadows by multiplying the shadow pixels by a constant. Then, it applies a filter to correct the edges. This method has one drawback which is the elimination of textured areas along with shadows, hence results in the lost of information. Therefore, inspired by Xiang's method, we develop a new shadow removal method that overcomes the previously described disadvantage. 


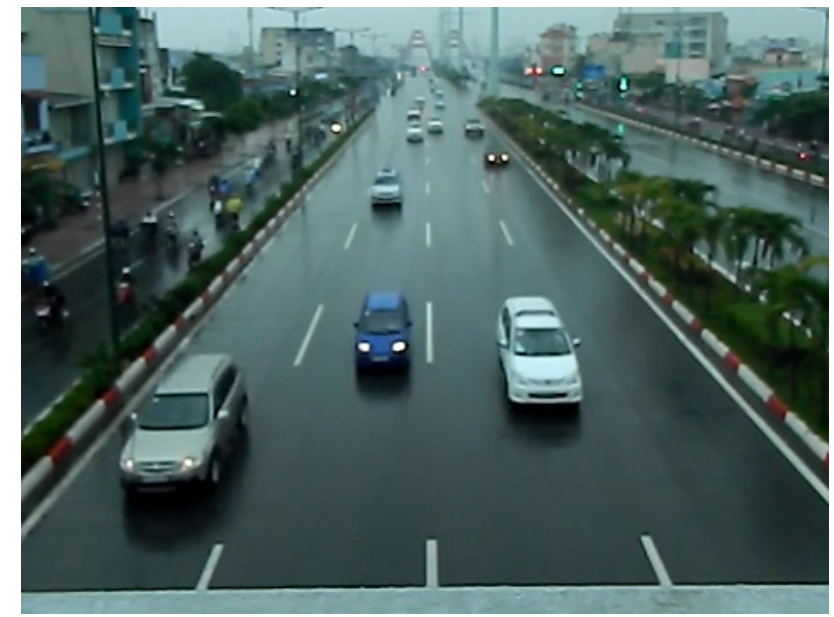

(a)

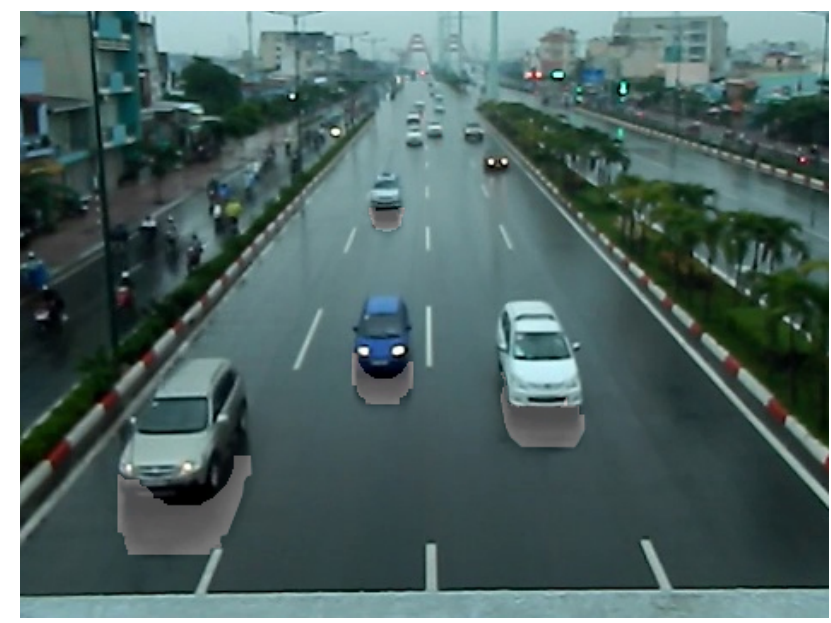

(c)

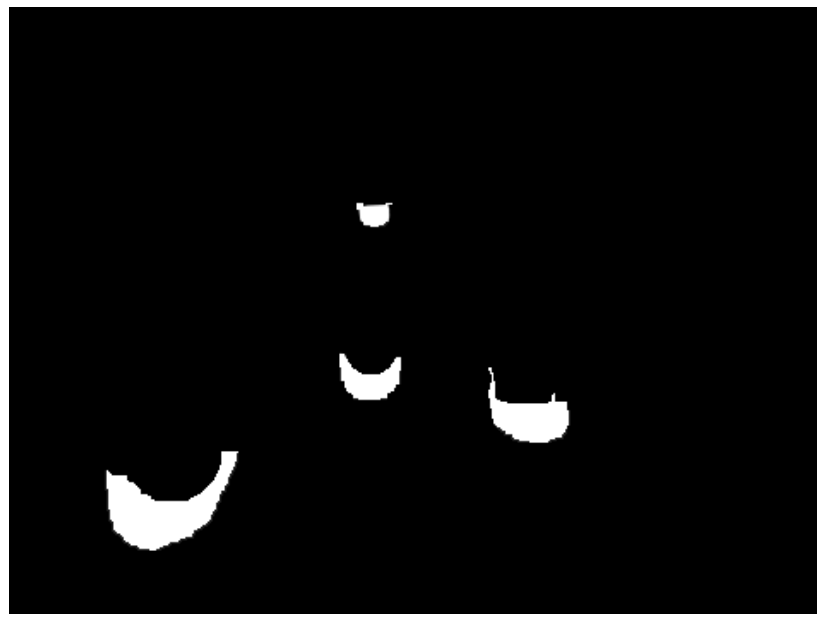

(b)

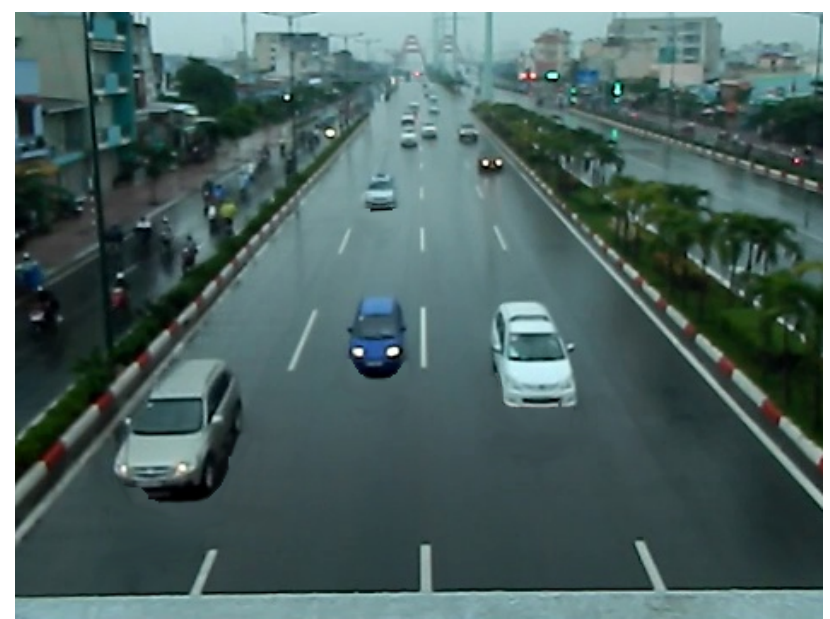

(d)

Figure 3. Results from reflection detection and removal algorithm. (a) original image, (b) detected reflected areas, (c) reflection regions and their neighbors, (d) reflection removal.

The proposed method is derived from an observation that the reflected areas have different intensities, in term of lightness, compared to non-reflected areas. In fact, the intensity of a reflection is gradually increased from dark to light. So, we can remove reflections by raising the lightness of these areas to slightly match with nonreflected ones while still preserving textures.

However, the intensity of non-reflected regions varies greatly. Hence, this poses a problem of determining a suitable intensity value to match with the reflected area. In order to scope with this problem, a meanshift filtering algorithm is applied on the $\mathrm{H}$ channel of the input image. The meanshift filtering segments the image into separated regions based on their colors. It also localizes each region with a uniform color intensity.

Usually, a reflected area is adjacent to one or more non-reflected areas. Let $R$ indicates the reflected area. Among all neighbors of $R$, the one that has the closest similarity in chromaticity ( $\mathrm{H}$ value) is chosen as the best-fit region. Then, the average chromaticity value among of the chosen segment is computed. Afterwards, we re-scale the reflected area chromaticity value to the computed value.

Still, there is one small issue that needs to be consid- ered. Changing the chromaticity values of the reflection surface often causes wrong colors. In this case, if the difference between the reflected area value and the bestfit region average value is larger than a certain threshold, the shadowed area value is not changed. Finally, all remaining edges are smoothed with a Gaussian mask. The result of this process can be shown in Figure 3(c).

\section{Headlight Reflection}

The problem of headlight reflection usually occurs in low lighting conditions, such as at dusk or nighttime. Figure 4 shows strong headlight reflection on the wet road surface that causes undesirable and challenging problems.

In common sense, the vehicle headlights possess the highest intensity value in the image. However, strong reflections on the wet road surface may have as high intensity value as the headlights, and hence greatly deteriorate the performance of traditional methods. This paper provides a headlight detection and removal method which consists of two steps.

Firstly, we identify the headlights' location. Based on the characteristic of vehicles' headlights, L channel is 


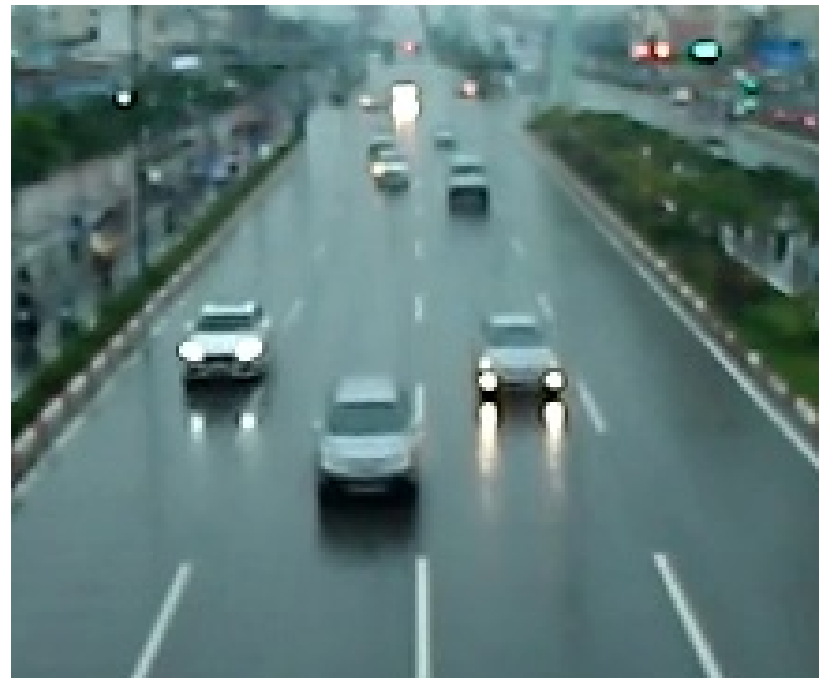

Figure 4. Example of vehicles with reflected headlight.

used to detect the high intensity lightness pixels. The locations of high intensity areas are easily determined by comparing them to a given threshold. During our study, we realize that any pixel that has an $\mathrm{L}$ value larger than 200 may suit our criteria of reflection. Then, each object is extracted from the image by determining its contour. The contour method is able to filter isolated objects or noises to achieve better blobs.

Secondly, we group any two objects by checking whether one is above the other with a reasonable amount of displacement. Each pair of objects will contain the headlights and their reflections of a single vehicle. Using the fact that reflection always appears below the headlight, we remove the lower object of each pair. Then, we use the remaining object as the seed to segment the actual vehicle.

However, there are some cases where two or more vehicles are following each other, which cause three or more objects are paired together. In this case, the headlight reflections of the back vehicles are covered by its front vehicles. So we only have to deal with the reflection placed in front of the first vehicle. We can simply reject the first front object and keep other back objects as separated vehicles. Figure 5(b) illustrates the results of this process.

\section{EXPERIMENT AND Discussion}

In order to evaluate the proposed method, we performed a number of experiments using images extracted from actual traffic surveillance videos. The videos were recorded at Pham Van Dong avenue (dataset PVD1), Ho Chi Minh city, Vietnam. All videos are of $480 \mathrm{p}$ resolution with the frame rate of $30 \mathrm{fps}$. Each testing video sequence includes vehicles of different colors. Since the cameras were set up on the top of the road, $[19,20]$ provide a method to locate the best observation zone of the road.

The final goal is to remove the reflections from vehicles and also eliminate their headlights. Experiments were set up to measure the accuracy of the reflection

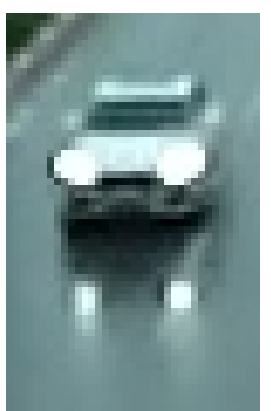

(a)

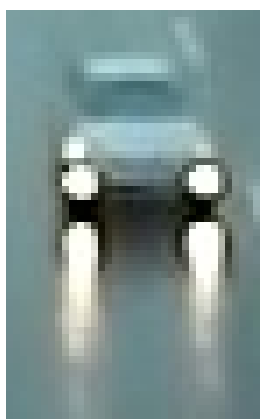

(d)

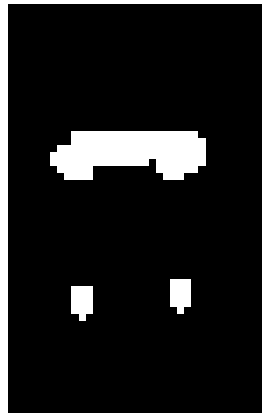

(b)

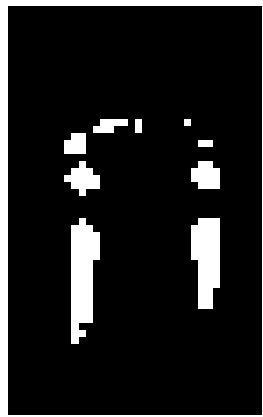

(e)

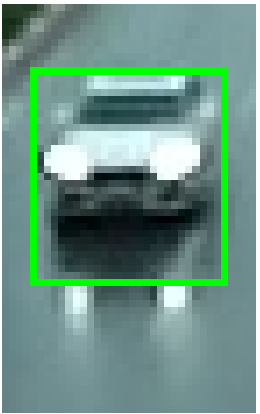

(c)

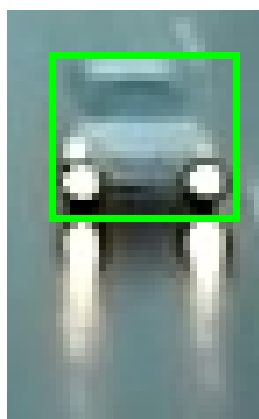

(f)
Figure 5. Headlight detection and removal process. From left to right: vehicles with reflected headlights, detected headlights and their reflections using $\mathrm{L}$ channel, reflection removal.

detection method, as well as the processing speed and computation time. We also compare our results with the ones from Murali et al. [13]. The first row of Figure 6 shows an experiment with reflections of a bus and a black car. Figure 6(c) is the result obtained using Murali's method in which the mirrors of vehicles were mistakenly classified as parts of reflections. Figure 6(d) shows the result of our method. Compare to Murali's result, ours method can clearly detect the reflected areas. In addition, the reflections detected in our result nearly resemble the ground truth. The second row of Figure 6 illustrates the results of experimenting on vehicle with headlight reflection. Once again, our method shows promising results.

Integration tests with vehicle classification algorithm mentioned in [20] were also performed. We compared the accuracy of vehicle classification and counting, as well as processing speed of the system with and without reflection detection method. Table III shows the results of vehicle counting. According to the results, the original vehicle classification algorithm mentioned in [20] has low accuracy. Meanwhile, our proposed method brought significant improvements to the original algorithm. Most of the small automobiles, such as cars and sedans, are detected and classified with an error rate of only $0.926 \%$. For large vehicles, the integrated system can still perform pretty well with only 2 misclassified cases. This can be achieved because our method eliminates the reflections which distort the shape and size of vehicles. Hence better measurements of each vehicle can be obtained.

Finally, we tested the performance of the proposed method in term of processing speed and overall com- 


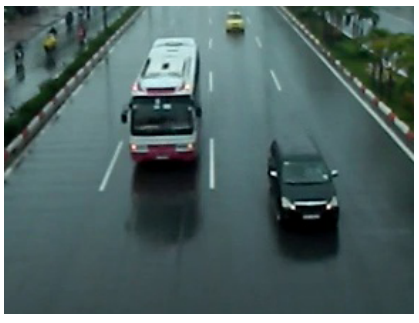

(a)

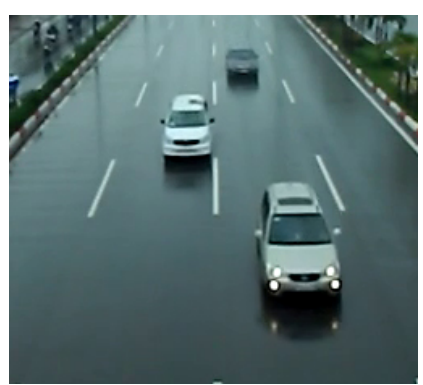

(e)

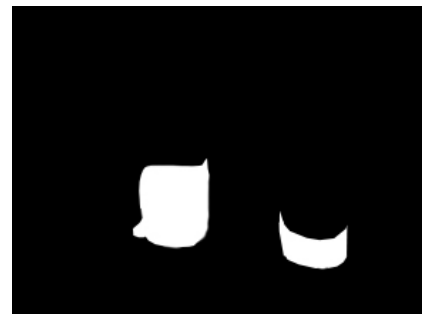

(b)

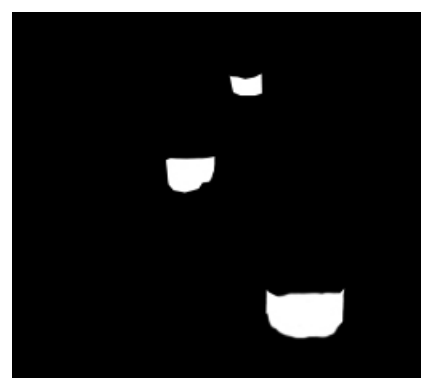

(f)

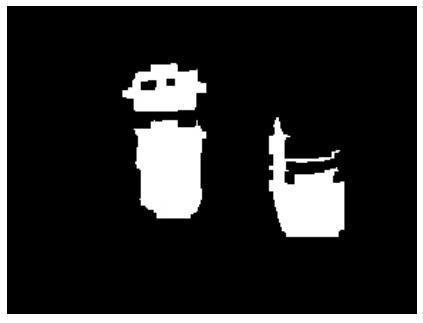

(c)

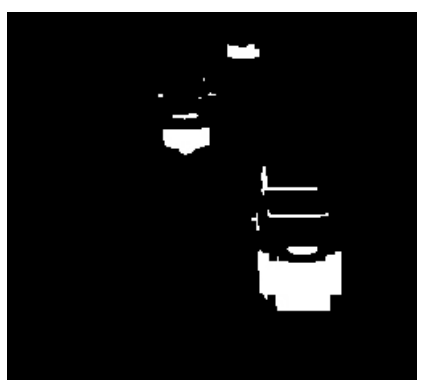

(g)

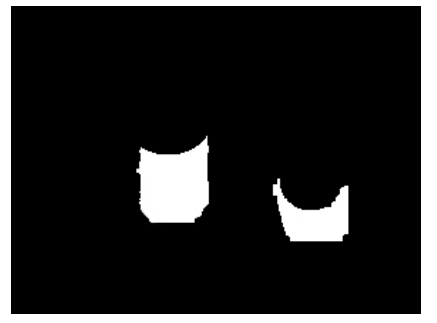

(d)

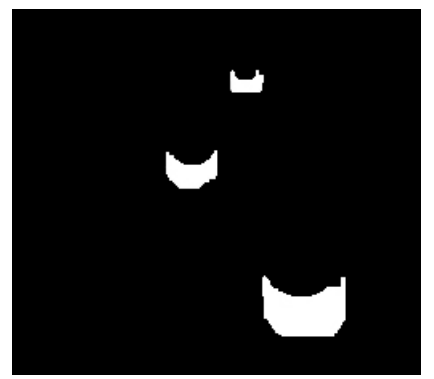

(h)

Figure 6. Experiments on reflection detection and removal. From left to right: original frame, reflection ground truth, Murali's method, our proposed method. First row: experiment on a bus with large reflection and a black car. Second row: experiment on vehicle with headlight reflection.

Table III

Comparison of Vehicle Classification Results between Ha's Method and the Proposed Method

\begin{tabular}{cccccccc}
\hline \multirow{2}{*}{ Class } & \multirow{2}{*}{ Number of vehicle } & \multicolumn{3}{c}{ Ha's method [20] } & \multicolumn{3}{c}{ The proposed method } \\
& & Counting & Error & Percentage Error & Counting & Error & Percentage Error \\
\hline Car + sedan & 108 & 84 & 24 & $22.22 \%$ & 107 & 1 & $0.926 \%$ \\
\hline Truck + bus & 10 & 15 & 5 & $50.00 \%$ & 12 & 2 & $20.00 \%$ \\
\hline
\end{tabular}

putation time. In our experiment, the proposed method took an average of 665.12 seconds to fully process 20000 frames. So the computation time for each frame is around 0.033256 seconds. In other words, the system can achieve real-time processing with the average frame rate of $30 \mathrm{fps}$.

\section{Conclusion}

In this paper, we presented a new method that can detect and remove reflections of vehicles in traffic surveillance videos. Experiments have shown that the proposed algorithm performs robustly under rainy conditions with different lighting intensities. Our proposed method first detects the reflected areas using $\mathrm{L}$ and $\mathrm{H}$ channels from LAB and HSV color spaces. Then, we segment these areas and re-scale the pixels to the average value of their nearest non-shadow neighbor. Finally, a Gaussian mask is applied to smooth the edges. The proposed method is also designed to deal with the cases of headlight reflection. By taking the advantage of the intensity differences between headlights and their reflections, we can easily detect and segment them. We notice the fact that on wet road surface reflections always reside under the headlights. We simply remove reflections by rejecting the lower segments. Several experiments have shown promising results with detecting and removing vehicle reflections.

\section{ACKNOWLEDGMENT}

This research was performed as a part of the "Improved Moving Vehicle Shadow Removal Algorithm for Traffic Monitoring System" project funded by the International University - Vietnam National University, Ho Chi Minh City.

\section{REFERENCES}

[1] R. Cucchiara, C. Crana, M. Piccardi, A. Prati, and S. Sirotti, "Improving shadow suppression in moving object detection with HSV color information," in IEEE Intelligent Transportation Systems, 2001, pp. 334-339.

[2] R. Cucchiara, C. Grana, M. Piccardi, and A. Prati, “Detecting objects, shadows and ghosts in video streams by exploiting color and motion information," in 11th International Conference on Image Analysis and Processing, 2001, pp. 360-365.

[3] R. Cucchiara, C. Grana, M. Piccardi, A. Prati, and M. Piccardi, "Detecting moving objects, ghosts, and shadows in video streams," IEEE Transactions on Pattern Analysis and Machine Intelligence, vol. 25, no. 10, pp. 1337-1342, Oct. 2003.

[4] C. Fredembach and S. Susstrunk, "Automatic and accurate shadow detection from (potentially) a single image using near-infrared information," Tech. Rep., 2010.

[5] J. Xiang, H. Fan, H. Liao, J. Xu, W. Sun, and S. Yu, "Moving Object Detection and Shadow Removing under Changing Illumination Condition," Mathematical Problems in Engineering, vol. 2014, pp. 1-10, 2014.

[6] Y.-P. Guan, "Adaptive Moving Cast Shadow Detection 
by Integrating Multiple Cues," Journal of Engineering, vol. 2014, no. 4, pp. 1-9, 2014.

[7] A. Sanin, C. Sanderson, and B. C. Lovell, "Improved Shadow Removal for Robust Person Tracking in Surveillance Scenarios," in 20th International Conference on Pattern Recognition, Aug. 2010, pp. 141-144.

[8] D. Conte, P. Foggia, G. Percannella, and M. Vento, "Removing Object Reflections in Videos by Global Optimization," IEEE Transactions on Circuits and Systems for Video Technology, vol. 22, no. 11, pp. 1623-1633, Nov. 2012.

[9] L. Xu, F. Qi, and R. Jiang, "Shadow Removal from a Single Image," in Sixth International Conference on Intelligent Systems Design and Applications, vol. 2, Oct. 2006, pp. 1049-1054.

[10] R. Cucchiara and M. Piccardi, "Vehicle Detection under Day and Night Illumination," ISCS-IIA99, 1999.

[11] W. Zhang, Q. M. J. Wu, and G. Wang, "Vehicle Headlights Detection Using Markov Random Fields," in Asian Conference on Computer Vision (ACCV 2009), 2009, pp. 169-179.

[12] I. Cabani, G. Toulminet, and A. Bensrhair, "Color-based detection of vehicle lights," in IEEE Proceedings. Intelligent Vehicles Symposium, vol. 2005, 2005, pp. 278-283.

[13] S. Murali and V. K. Govindan, "Shadow Detection and Removal from a Single Image Using LAB Color Space," Cybernetics and Information Technologies, vol. 13, no. 1, pp. 95-103, Jan. 2013.

[14] A. Suny and N. Mithila, "A Shadow Detection and Removal from a Single Image Using LAB Color Space," International Journal of Computer Science Issues, vol. 10, no. 4, pp. 270-273, Jan. 2013.

[15] N. Salamati, A. Germain, and S. Susstrunk, "Removing shadows from images using color and near-infrared," IEEE International Conference On Image Processing, pp. 1753-1756, 2011

[16] T. P. Nguyen, D. N.-N. Tran, T. K. Huynh, and S. V.-U. $\mathrm{Ha}$, "Disorder detection approach to background modeling in traffic surveillance system," Journal of Science and Technology, Vietnamese Academy of Science and Technology, vol. 52, no. 4A, pp. 140-149, 2014.

[17] S. V.-U. Ha, H.-H. Nguyen, T. K. Huynh, and P. HoThanh, "Lane Detection in Intelligent Traffic System Using Probabilistic Model," in 16th Asia Pacific Industrial Engineering \& Management Systems Conference (APIEMS 2015), 2015, pp. 1856-1863.

[18] S. V.-U. Ha, H.-H. Nguyen, H. M. Tran, and P. Ho-Thanh, "Improved optical flow estimation in wrong way vehicle detection," Journal of Information Assurance and Security, vol. 9, no. 5, pp. 165-169, Dec. 2014.

[19] S. V.-U. Ha, L. H. Pham, H. M. Tran, and P. Ho-Thanh, "Improved vehicles detection \& classification algorithm for traffic surveillance system," Journal of Information Assurance and Security, vol. 9, no. 5, pp. 268-277, 2014.

[20] S. V.-U. Ha, L. H. Pham, H. N. Phan, and P. Ho-Thanh, "A Robust Algorithm for Vehicle Detection and Classification in Intelligent Traffic System," in 16th Asia Pacific Industrial Engineering \& Management Systems Conference (APIEMS 2015), no. 1, 2015, pp. 1832-1838.

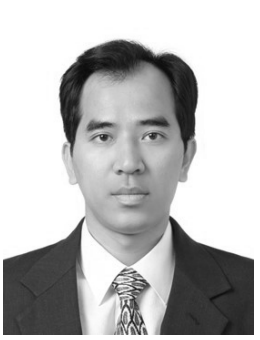

Synh Viet Uyen Ha is a lecturer at the School of Computer Science and Engineering, International University, Vietnam National University, Ho Chi Minh City. He received an engineer degree from Ho Chi Minh City University of Technology, Vietnam in 1996, and a M.Sc. degree from Ho Chi Minh University of Natural Science, Vietnam National University, Ho Chi Minh City, in 1999, both in computer science and engineering. He received a Ph.D. degree from School of Information and Communication Engineering, Sungkyunkwan University, Suwon, Korea in 2010. His research interests include machine learning, computer vision and image processing.

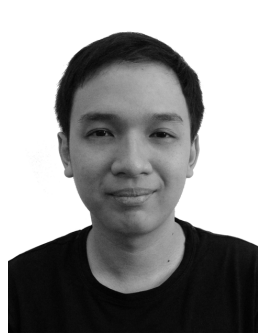

Nhan Than Pham is a senior student and is completing a B.Sc. degree in computer science and engineering from International University, Vietnam National University, Ho Chi Minh City, Vietnam. He has been doing research related to image processing and computer vision under the supervision of Dr. Synh Viet-Uyen Ha. His research interests include image processing, computer vision.

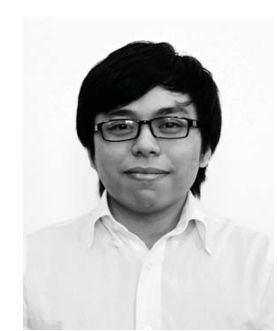

Long Hoang Pham is a master student and is completing a M.S. degree in information technology management from International University, Vietnam National University, Ho Chi Minh City, Vietnam. He has been doing research related to image processing and computer vision under the supervision of Dr. Synh Viet-Uyen Ha. His research interests include image processing, computer vision, artificial intelligence and robotics.

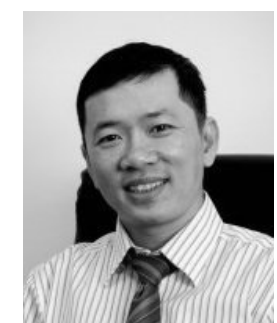

Ha Manh Tran is a lecturer of computer science at International University, Vietnam National University, Ho Chi Minh City, Vietnam. He received his master degree in 2004 from the University of Birmingham, United Kingdom and his doctoral degree in 2009 from Jacobs University Bremen, Germany. His research interests include network management, distributed computing, big data analytics. 